\title{
Development of the integration of pharmaceutical science and pharmacy practice through a new model of Pharmacy Practice Skills (PPS) practical classes
}

Author: Jon Waterfield, De Montfort University, Leicester

Background: Third year MPharm undergraduate classes in a 'Skills for Practice' module at this institution have moved to a common format that focus on four key skill areas: information gathering, information giving, teamwork and assessing the work of others. Each Pharmacy Practice Skills (PPS) class is based on a specific therapeutic area where the main aim is to facilitate integration across modules using a common skills-based format.

Description of work: All sessions are designed for 20 students and each subgroup of 5 students rotates around four timed workstations. For example a PPS session on the respiratory system includes:

- A systematic process of gathering information when a patient requests an emergency supply of an inhaler (WS1)

- The accurate and bespoke provision of information when counselling a patient with adherence issues (WS2)

- Interaction with colleagues utilising a range of resources to apply knowledge of COPD (WS3)

- Checking the work of others including legal and clinical checks (WS4).

This session fosters integration across all streams of the programme:

- Practice and Healthcare

- Body Systems and Clinical Studies

- Pharmaceutical Sciences

Proposed evaluation: Current evaluation of this work has been mainly through student and staff feedback. Further work is proposed using a mixed methods study to evaluate student views on how PPS sessions facilitate the integration of pharmaceutical science and pharmacy practice. The purpose of this work is to gain some insight into how students create connections between knowledge within different modules. This work will support our understanding of how students apply different types of knowledge to generic practice skills. This evaluation will be used in the ongoing development and further integration of the MPharm programme. 